\title{
Lipid structure does not modify incorporation of EPA and DHA into blood lipids in healthy adults: a randomised-controlled trial
}

\author{
Annette L. West ${ }^{1}$, Graham C. Burdge ${ }^{1 *}$ and Philip C. Calder ${ }^{1,2}$ \\ ${ }^{1}$ Academic Unit of Human Development and Health, University of Southampton, Southampton SO16 6YD, UK \\ ${ }^{2}$ National Institute for Health Research Southampton Biomedical Research Centre, University Hospital Southampton National \\ Health Service Foundation Trust, University of Southampton, Southampton SO16 6YD, UK
}

(Submitted 16 May 2016 - Final revision received 16 June 2016 - Accepted 21 June 2016 - First published online 18 July 2016)

\section{Abstract}

Dietary supplementation is an effective means to improve EPA and DHA status. However, it is unclear whether lipid structure affects EPA + DHA bioavailability. We determined the effect of consuming different EPA and DHA lipid structures on their concentrations in blood during the postprandial period and during dietary supplementation compared with unmodified fish oil TAG (uTAG). In a postprandial crossover study, healthy men $(n 9)$ consumed in random order test meals containing $1.1 \mathrm{~g}$ EPA $+0 \cdot 37 \mathrm{~g}$ DHA as either uTAG, re-esterified TAG, free fatty acids (FFA) or ethyl esters (EE). In a parallel design supplementation study, healthy men and women ( $n$ 10/sex per supplement) consumed one supplement type for 12 weeks. Fatty acid composition was determined by GC. EPA incorporation over $6 \mathrm{~h}$ into TAG or phosphatidylcholine (PC) did not differ between lipid structures. EPA enrichment in NEFA was lower from EE than from uTAG $(P=0 \cdot 01)$. Plasma TAG, PC or NEFA DHA incorporation did not differ between lipid structures. Lipid structure did not affect TAG or NEFA EPA incorporation and PC or NEFA DHA incorporation following dietary supplementation. Plasma TAG peak DHA incorporation was greater $(P=0.02)$ and time to peak shorter $(P=0.02)$ from FFA than from uTAG in men. In both studies, the order of EPA and DHA incorporation was PC $>$ TAG $>$ NEFA. In conclusion, EPA and DHA lipid structure may not be an important consideration in dietary interventions.

Key words: EPA: DHA: Postprandial: Lipid structure: PUFA

Dietary supplementation with EPA $(20: 5 n-3)$ and DHA (22:6n-3) confers well-established changes in the biophysical properties of cell membranes and cell signalling processes that are associated with positive effects on health ${ }^{(1)}$. EPA and DHA are consumed in the diet primarily as components of the muscle of oily fish. Consequently, several organisations have published recommendations for oily fish consumption in order to provide sufficient EPA + DHA for health benefits ${ }^{(2)}$. However, low levels of oily fish consumption in some populations have limited their effectiveness $^{(3)}$. Dietary supplementation with encapsulated oils containing EPA+DHA provides an alternative means of increasing their intake ${ }^{(4)}$, which may be facilitated by highly purified EPA + DHA preparations that reduce the volume of oil required to achieve health benefits ${ }^{(4)}$. It is, therefore, important to identify the preparations that are most effective in increasing $\mathrm{EPA}+\mathrm{DHA}$ status

The lipid structures of EPA + DHA used most commonly in dietary supplements are unmodified fish oil TAG (UTAG) from fish body oil or cod liver oil, re-esterified TAG (rTAG), krill oil phospholipid, free fatty acids (FFA) and ethyl esters (EE). Differences in the lipid structure in which EPA+DHA are ingested may influence their bioavailability and accumulation within lipid pools. The rank order of the increment in EPA + DHA status after consuming $3 \cdot 3 \mathrm{~g}$ EPA + DHA daily for 2 weeks in men and women was rTAG $>$ fish body oil $>$ FFA $>$ cod liver oil $>\mathrm{EE}^{(4)}$. Similarly, the increment in omega- 3 index ${ }^{(5)}$ was greater when EPA+DHA (1.68 g daily) were consumed for 6 months as rTAG compared with EE in moderately hypertriacylglycerolaemic subjects $^{(6,7)}$. EPA incorporation into plasma TAG over $24 \mathrm{~h}$ has been shown to increase when consumed as $s n-2$ monoacylglycerol, TAG or FFA compared with $\mathrm{EE}^{(8,9)}$. Postprandial incorporation of EPA+DHA into plasma TAG was greater when ingested as FFA than as TAG (approximately 38\%) or EE $(80 \%)^{(10,11)}$. In addition, the bioavailability of EPA from rTAG was shown to be greater when compared with $s n$-2 monoacylglycerol $^{(12)}$, although others have not found this result ${ }^{(13,14)}$. EPA and DHA bioavailability may also be influenced by the total fat composition of a meal ${ }^{(11,15)}$. Furthermore, the magnitude of EPA and DHA accumulation appears to differ between plasma lipid classes: phospholipids > cholesteryl esters $>\mathrm{TAG}^{(2)}$.

Differences in study design have produced uncertainty in the literature about the effects of lipid structure on EPA and DHA bioavailability during dietary supplementation. The purpose of this study was to compare directly the effects of different lipid

Abbreviations: EE, ethyl esters; FFA, free fatty acids; iAUC, incremental AUC; PC, phosphatidylcholine; rTAG, re-esterified TAG; UTAG, unmodified fish oil TAG. 
preparations of $\mathrm{EPA}+\mathrm{DHA}$ in increasing their concentrations in blood lipids. We measured EPA and DHA concentrations in plasma TAG, NEFA and phosphatidylcholine (PC) after consumption of EPA+DHA as rTAG, FFA or EE in a single meal and compared this with UTAG that is used commonly in commercial dietary supplements. We then investigated whether lipid structure altered EPA and DHA incorporation into blood after daily consumption. We also assessed the effect of enteric-protective capsules containing rTAG on postprandial EPA + DHA incorporation into blood lipids and of sex and BMI on EPA + DHA assimilation during 12 weeks of dietary supplementation.

\section{Methods}

\section{Ethics approval and study registration}

The present study was conducted in accordance with the declaration of Helsinki. The postprandial study was approved by the Isle of Wight, Portsmouth and South East Hampshire Research Ethics Committee B (approval no. 09/H0501/98), and the dietary supplementation study was approved by the Southampton and South West Hampshire Research Ethics Committee B (approval no. 11/SC/0049). The postprandial study is registered as ISRCTN11656280, and the dietary supplementation study is registered as ISRCTN46532656 at www.controlled-trials.com.

\section{Postprandial study: participants, design, sample collection and specimen processing}

In all, twelve volunteers showed initial interest in the study; two of them did not complete the screening process. Of the ten participants who completed the study, one was subsequently found to have elevated fasting blood glucose concentration $(>7 \mathrm{mmol} / \mathrm{l})$ and the data were not included in the final analyses (online Supplementary Fig. S1). The participants of the postprandial study included nine healthy men with a median age of 26 (range 22-38) years, BMI of 24 (range $20-30$ ) $\mathrm{kg} / \mathrm{m}^{2}$ and fasting plasma TAG of 0.8 (range $0 \cdot 4-2 \cdot 2$ ) $\mathrm{mmol} / \mathrm{l}$, NEFA of 0.3 (range $0 \cdot 2-0 \cdot 9$ ) $\mathrm{mmol} / 1$ and glucose concentration of 5.3 (range $4.5-6.0) \mathrm{mmol} / \mathrm{l}$. All included participants self-reported that they were low consumers of oily fish ( $<1$ meal/week) and that they did not use fish oil supplements. The double-blinded, postprandial study was based on our previous single-blinded, cross-over design ${ }^{(16)}$. Each participant took part in 5 postprandial study $\mathrm{d}$ in random order, with an interval of at least $14 \mathrm{~d}$ between each study day. Capsule containers were labelled with anonymised codes, and the order in which the participants took the supplements was assigned using a random number generator (Random.org) by an independent member of the staff. The nine participants consumed their habitual diet throughout the study. On the day preceding any postprandial study day, participants were asked to consume their evening meal by 21.00 hours and to fast until the postprandial study commenced. Participants arrived at the National Institute for Health Research Wellcome Trust Clinical Research Facility, Southampton General Hospital, Southampton, UK, at approximately 07.00 hours. A cannula was inserted into a forearm vein, and a baseline blood sample $(5 \mathrm{ml})$ was collected into an evacuated tube containing heparin sulphate as anticoagulant.
Participants consumed the same test meal on each occasion. This contained $4.3 \mathrm{MJ}$ total energy derived from carbohydrate $(117 \mathrm{~g})$, protein $(15 \mathrm{~g})$ and a blend of fats $(55 \mathrm{~g})$ from sunflower oil, double cream, flaxseed oil and olive oil, which provided a fatty acid pattern that was representative of the typical UK diet as described previously ${ }^{(16)}$. The fat and protein components of the test meal were administered as an emulsion (total volume made up to $160 \mathrm{ml}$ with water). The carbohydrate component of the meal was toast with marmalade. Participants consumed the test meal within $15 \mathrm{~min}$. On each postprandial study day, participants consumed one of the following supplements: gelatin-encapsulated UTAG, rTAG, EE or FFA each of which provided approximately EPA $1 \cdot 1 \mathrm{~g}+$ DHA $0.37 \mathrm{~g}$ (Table 1). The rTAG, EE and FFA preparations contained less SFA and MUFA and 18:2n-6 compared with uTAG (Table 1). In order to determine whether exposure to the gastric environment affected the bioavailability of EPA and DHA, on one occasion participants consumed rTAG (providing EPA $1 \cdot 1 \mathrm{~g}+$ DHA $0.37 \mathrm{~g}$ ) encapsulated in an enteric-resistant coating. Blood samples $(5 \mathrm{ml})$ were collected at $0 \cdot 5,1,1 \cdot 5,2,2 \cdot 5,3,4,5$ and $6 \mathrm{~h}$ after consumption of the test meal. Participants remained at rest and were allowed free access to water throughout the study. Plasma was isolated form blood samples by centrifugation and stored at $-80^{\circ} \mathrm{C}^{(16)}$.

\section{Supplementation study design, sample collection and specimen processing}

The supplementation study had a double-blinded, parallel design. In total, 200 volunteers enquired about the study,

Table 1. Fatty acid compositions of the lipid supplements

\begin{tabular}{lrrrrr}
\hline & \multicolumn{5}{c}{ Intake of fatty acids from supplements } \\
& \multicolumn{5}{c}{ per test meal or per day (mg) } \\
\cline { 2 - 6 } & FFA & UTAG & rTAG(EC) & EE & rTAG \\
\hline $14: 0$ & 0 & 355 & 0 & 0 & 0 \\
$16: 0$ & 6 & 785 & 6 & 5 & 6 \\
$16: 1 n-7$ & 2 & 425 & 2 & 2 & 2 \\
$18: 0$ & 2 & 151 & 27 & 1 & 27 \\
$18: 1 n-9$ & 44 & 483 & 63 & 40 & 63 \\
$18: 1 n-7$ & 3 & 143 & 17 & 7 & 19 \\
Unknown & 7 & 164 & 0 & 3 & 0 \\
$18: 2 n-6$ & 289 & 1030 & 165 & 261 & 165 \\
$18: 3 n-6$ & 188 & 144 & 127 & 170 & 127 \\
$18: 3 n-3$ & 8 & 48 & 12 & 7 & 12 \\
$20: 0$ & 1 & 4 & 0 & 1 & 1 \\
$20: 1 n-9$ & 64 & 182 & 26 & 59 & 26 \\
$20: 2 n-6$ & 3 & 12 & 5 & 3 & 5 \\
$20: 3 n-6$ & 4 & 9 & 5 & 4 & 5 \\
$20: 4 n-6$ & 68 & 72 & 51 & 60 & 50 \\
$22: 0$ & 3 & 0 & 0 & 3 & 0 \\
$20: 4 n-3$ & 30 & 59 & 42 & 27 & 41 \\
EPA & 1284 & 1391 & 1199 & 1159 & 1190 \\
$24: 0$ & 31 & 47 & 54 & 27 & 53 \\
$24: 1 n-9$ & 10 & 13 & 14 & 9 & 13 \\
$22: 5 n-3$ & 37 & 104 & 70 & 33 & 70 \\
DHA & 397 & 379 & 384 & 355 & 394 \\
EPA + DHA & 1681 & 1771 & 1583 & 1514 & 1584 \\
Peroxide value (mmol/kg) & $<0 \cdot 9$ & $<0 \cdot 6$ & $<2 \cdot 1$ & $<0 \cdot 9$ & $<2 \cdot 1$ \\
\hline & & & & \\
& & & &
\end{tabular}

FFA, free fatty acid; UTAG, unmodified TAG; rTAG(EC), enteric-protected re-esterified TAG; EE, ethyl ester; rTAG, re-esterified TAG; ppm, parts per million.

* Concentrations of contaminants: heavy metals $(\mathrm{Cd}, \mathrm{Hg}, \mathrm{Pb}, \mathrm{As})<0.1 \mathrm{ppm}$; polychlorinated biphenyls $<0.09 \mathrm{ppm}$; dioxins $<1.5 \mathrm{pg}$ Teq/g; pesticides $<1 \mathrm{ppm}$. 
of which 120 agreed to undergo initial screening. Of these, twenty subsequently either declined further participation or were found to be ineligible for reasons not covered in the questionnaire, and a further twenty participants were randomised to an arm of the study that was not included in the analysis design (online Supplementary Fig. S2). These participants consumed capsules of medium-chain TAG from palm oil. As the palm oil did not contain EPA or DHA, this arm of the study was excluded from the final analysis because it did not provide a direct comparator for the other lipid structures. In all, eighty participants (forty men and forty women) were randomised to receive one of the four supplements that contained EPA and DHA (online Supplementary Fig. S2); two participants withdrew during the study period because they found taking capsules unpleasant and one more was unable to attend the appointments.

The participants who completed the supplementation study were men aged between 19 and 38 years with BMI of $20-30 \mathrm{~kg} / \mathrm{m}^{2}$ and women aged between 19 and 44 years with BMI of $20-31 \mathrm{~kg} / \mathrm{m}^{2}$ who were in good general health, who did not consume fish oil or other oil supplements and did not eat more than one portion of oily fishmeal per week (Table 2 ). There were no significant differences within each sex in age, BMI or in fasting plasma TAG or NEFA concentrations between supplement groups. Participants consumed their habitual diet throughout the study. At study entry, participants were asked to fast from 21.00 hours until after a blood sample $(20 \mathrm{ml})$ was collected between approximately 08.00 and 10.00 hours. Participants ( $n$ 10/sex per supplement) were then assigned at random to consume one of four dietary supplements used in the postprandial study for 12 weeks. The identity of the supplements was anonymised, and the allocation of the participants was randomised using a random number generator by the manufacturer of the supplements (Vifor Pharma Ltd). The supplements were gelatin-encapsulated UTAG, EE or FFA or rTAG-encapsulated in an enteric-resistant coating, each of which provided approximately $1.1 \mathrm{~g} / \mathrm{d}$ of EPA and $0.35 \mathrm{~g} / \mathrm{d}$ of DHA. The gelatinencapsulated rTAG dietary supplement was omitted from the supplementation study because of lack of differences in EPA and DHA incorporation into blood lipids between coating materials in the postprandial study. Fasting blood samples $(20 \mathrm{ml})$ were collected during the supplementation period after $1,2,4,8$ and 12 weeks. Plasma was isolated from blood samples by centrifugation and stored at $-80^{\circ} \mathrm{C}^{(16)}$.

\section{Analysis of plasma lipid fatty acid composition}

The fatty acid composition of plasma TAG, NEFA and PC was determined by $\mathrm{GC}^{(16)}$. In brief, internal standards (dipentadecanoyl PC $(100 \mu \mathrm{g})$, tripentadecanoin $(100 \mu \mathrm{g})$ and heneicosanoic acid $(50 \mu \mathrm{g}))$ were added, and total lipids were extracted from plasma $(0.8 \mathrm{ml})$ with chloroform and methanol ${ }^{(17)}$ and dried under $\mathrm{N}_{2}$. The total lipid extracts were dissolved in chloroform, and individual lipid classes were then isolated by solid-phase extraction using Bond Elut 100-mg aminopropylsilica cartridges (Agilent Technologies) ${ }^{(18)}$. The purified lipid classes were dissolved in toluene, and fatty acid methyl esters (FAME) were synthesised by heating the purified lipids at $50^{\circ} \mathrm{C}$

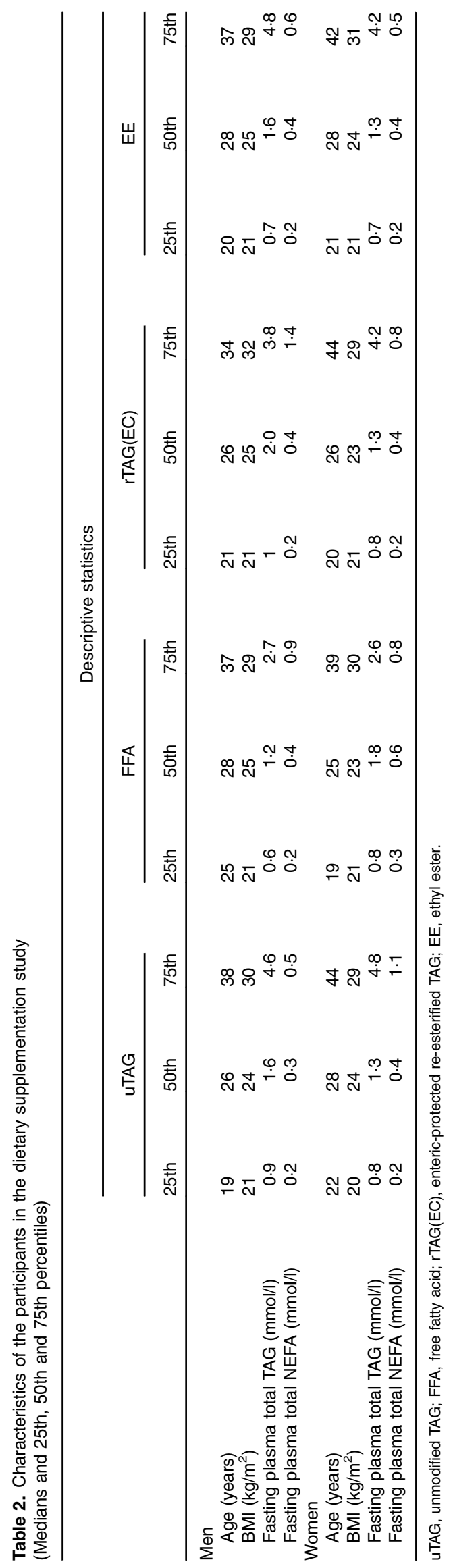


in the presence of methanol containing $2 \%(\mathrm{v} / \mathrm{v})$ sulphuric $\operatorname{acid}^{(18)}$. FAME were recovered by extraction with hexane and resolved in a BPX-70 fused silica capillary column $(32 \mathrm{~m} \times$ $0.25 \mathrm{~mm} \times 25 \mu \mathrm{m}$; SGE Analytical Science) using an Agilent 6890 gas chromatograph equipped with flame ionisation detection (Agilent Technologies Ltd) ${ }^{(16)}$. The concentrations of EPA and DHA in blood lipid classes, expressed as $\mu \mathrm{mol} / \mathrm{l}$, were calculated from the ratio of their peak area:the internal standard, multiplied by the amount of standard and corrected for the volume of plasma extracted.

\section{Statistical analysis}

The researchers, participants and staff involved in care of the participants (e.g. research nurses) were blinded to the allocation of supplements until data analysis had been completed. Data were analysed using SPSS version 21 (SPSS Inc.). The data sets were not distributed normally and did not approximate a normal distribution after log-transformation. Therefore, nonparametric analyses were used throughout. Comparison of data sets involving repeated measures was by Friedman's test with comparisons between groups at specific time points by Wilcoxon's signed-rank test. Comparison of single time point measurements between groups of different subjects was by the Kruskal-Wallis test or the Mann-Whitney $U$ test. Comparison of baseline and end-of-study total lipid concentrations in the supplementation study was by Wilcoxon's signed-rank test.

For all comparisons, the gelatin-coated UTAG was used as the reference data set. As we intended to model the effect of lipid structure in a manner relevant to the general population, the amount of EPA and DHA consumed by each participant was not adjusted for BMI. As the data were not normally distributed, it was not possible to carry out analysis of co-variance. However, we assessed the relationship between the incremental AUC (iAUC) for EPA and DHA and BMI using Spearman's rank order correlation test. Age has been shown to be related to incorporation of EPA and $\mathrm{DHA}^{(19)}$. Therefore, the relationship between age and the iAUC for EPA and DHA was also assessed by Spearman's rank order correlation test.

The postprandial study was powered according to the anticipated change in EPA content of plasma TAG. On the basis of our previous study ${ }^{(16)}$, a sample size of nine would have given a statistical power of $85 \%$ power to detect an increase in plasma TAG EPA concentration of $15 \mu \mathrm{mol} / \mathrm{l}$ at $4 \mathrm{~h}$ with a probability of $<0 \cdot 05$. The dietary supplementation calculations were based on the anticipated change in plasma PC EPA from $1.7 \%$ of total fatty acids to $2.9 \%{ }^{(20)}$. Consequently, a sample size of ten was expected to provide $90 \%$ statistical power for detecting this increment in plasma PC EPA concentration with a probability of $P<0.05$.

\section{Results}

\section{Postprandial incorporation of EPA and DHA into blood} lipids

There were no significant differences between supplements in the concentrations of EPA or DHA at baseline (online Supplementary Table S1). The changes in the concentrations of
EPA and DHA in plasma TAG, NEFA and PC are summarised in the online Supplementary Fig. S3 and Table S3. There were no significant differences between supplements in the iAUC, maximum concentration $\left(C_{\max }\right)$ or time to $C_{\max }\left(T_{\max }\right)$ for incorporation of EPA into plasma TAG or PC (Table 3 ). There were no significant differences between supplements in iAUC or $T_{\max }$ for incorporation of EPA into plasma NEFA (Table 3). However, $C_{\max }$ for the incorporation of EPA into plasma NEFA differed significantly $\left(\chi^{2}(4, n 16)=12 \cdot 1, P=0 \cdot 02\right)$ between groups (Table 3 ). Pairwise testing showed that the $C_{\max }$ for $\mathrm{EE}$ preparation, but not the other molecular structures, was lower than that for uTAG $(Z=-2.549, P=0.011)$ with a large effect size $(r-0 \cdot 9)$.

There were no significant differences between supplements in iAUC or $C_{\max }$ for incorporation of DHA into plasma TAG or PC (Table 3). However, $T_{\max }$ for the incorporation of DHA into plasma PC differed significantly $\left(\chi^{2}(4, n 18)=11 \cdot 1, P=0 \cdot 03\right)$ between groups (Table 2), although this was not supported by pairwise testing between groups (Wilcoxon's signed-rank test). There were no significant differences between supplements in iAUC, $T_{\max }$ or $C_{\max }$ for incorporation of DHA into plasma NEFA (Table 3).

Incorporation of EPA and DHA during the postprandial period differed between plasma lipid classes such that the rank order of EPA iAUC was PC $>$ TAG $>$ NEFA and of EPA $C_{\max }$ was TAG $>$ PC $>$ NEFA, irrespective of the structure of the supplement (Table 3, online Supplementary Table S2). There was no statistically significant difference in EPA $T_{\max }$ among lipid classes (Table 3). The rank order of DHA iAUC and $C_{\max }$ was PC $>$ TAG $>$ NEFA and of DHA $T_{\max }$ was NEFA $>$ TAG $>$ PC, irrespective of the structure of the supplement (Table 3, online Supplementary Table S2).

There was a significant positive association between incorporation of EPA from rTAG(EC) into plasma PC and BMI (online Supplementary Table S3). There was a significant negative association between incorporation of EPA from rTAG into plasma PC and BMI (online Supplementary Table S3). There was a significant negative association between incorporation of EPA EE into plasma PC and age (online Supplementary Table S3). There was a significant positive association between incorporation of DHA from rTAG(EC) into plasma PC and age (online Supplementary Table S3). There were no other significant associations between BMI or age and incorporation of EPA and DHA into plasma lipids (online Supplementary Table S3).

\section{Incorporation of EPA and DHA into blood lipids during dietary supplementation}

There were no significant differences between the groups in the concentrations of EPA or DHA at baseline (online Supplementary Table S1). There were no significant changes in the concentrations of total plasma TAG, PC or NEFA between the start and the end of the supplementation study (online Supplementary Table S4). The changes in EPA and DHA concentrations in plasma lipids during 12 weeks of supplementation in men and women are summarised in the online Supplementary Fig. S4 and S5, and in Tables 4 and 5, respectively. There were 
Table 3. Postprandial changes in EPA and DHA concentrations in plasma TAG, phosphatidylcholine (PC) and NEFA in healthy men (Medians and 25th, 50th and 75th percentiles; $n$ 9/group)

\begin{tabular}{|c|c|c|c|c|c|c|c|c|c|c|c|c|c|c|c|}
\hline \multirow[b]{2}{*}{ Lipid structure of supplements } & \multicolumn{3}{|c|}{ UTAG } & \multicolumn{3}{|c|}{ FFA } & \multicolumn{3}{|c|}{ rTAG(EC) } & \multicolumn{3}{|c|}{ EE } & \multicolumn{3}{|c|}{ rTAG } \\
\hline & 25th & 50th & 75th & 25th & 50th & 75th & 25th & 50th & 75th & 25th & 50th & 75th & 25th & 50th & 75th \\
\hline \multicolumn{16}{|l|}{ Plasma lipid class (parameter) } \\
\hline \multicolumn{16}{|l|}{ TAG } \\
\hline EPA iAUC ( $\mu \mathrm{mol} / \mathrm{h}$ per litre) & 140 & 230 & 328 & 140 & 226 & 462 & 172 & 216 & 293 & 151 & 202 & 276 & 150 & 183 & 432 \\
\hline EPA $C_{\max }(\mu \mathrm{mol} / \mathrm{l})$ & 22 & 51 & 75 & 20 & 76 & 173 & 32 & 48 & 82 & 30 & 53 & 67 & 25 & 38 & 119 \\
\hline EPA $T_{\max }(\mathrm{h})$ & 3 & 4 & 5 & 3 & 5 & 6 & 4 & 5 & 5 & 3 & 5 & 6 & 3 & 4 & 6 \\
\hline DHA iAUC ( $\mu \mathrm{mol} / \mathrm{h}$ per litre) & 484 & 499 & 527 & 486 & 513 & 575 & 491 & 514 & 535 & 479 & 503 & 523 & 484 & 515 & 578 \\
\hline DHA $C_{\max }(\mu \mathrm{mol} / \mathrm{l})$ & 11 & 17 & 26 & 9 & 23 & 54 & 10 & 22 & 32 & 10 & 13 & 22 & 9 & 16 & 42 \\
\hline DHA $T_{\max }(\mathrm{h})$ & 2 & 3 & 4 & 3 & 3 & 5 & 4 & 5 & 5 & 2 & 4 & 6 & 3 & 4 & 5 \\
\hline \multicolumn{16}{|l|}{ PC } \\
\hline EPA iAUC ( $\mu \mathrm{mol} / \mathrm{h}$ per litre) & 335 & 359 & 501 & 310 & 370 & 401 & 330 & 361 & 431 & 346 & 386 & 440 & 321 & 368 & 417 \\
\hline $\operatorname{EPA} C_{\max }(\mu \mathrm{mol} / \mathrm{l})$ & 11 & 18 & 52 & 11 & 20 & 33 & 15 & 21 & 37 & 14 & 31 & 37 & 15 & 21 & 35 \\
\hline EPA $T_{\max }(\mathrm{h})$ & 3 & 4 & 6 & 3 & 5 & 5 & 3 & 5 & 6 & 5 & 5 & 6 & 4 & 5 & 6 \\
\hline DHA iAUC $(\mu \mathrm{mol} / \mathrm{h}$ per litre $)$ & 484 & 589 & 686 & 471 & 541 & 580 & 473 & 551 & 608 & 427 & 540 & 616 & 511 & 584 & 714 \\
\hline DHA $C_{\max }(\mu \mathrm{mol} / \mathrm{l})$ & 26 & 31 & 53 & 21 & 26 & 37 & 14 & 32 & 70 & 22 & 27 & 57 & 17 & 41 & 78 \\
\hline $\mathrm{DHA} T_{\max }(\mathrm{h})$ & 3 & 4 & 4 & 1 & 1 & 3 & 2 & 2 & 4 & 1 & 2 & 3 & 3 & 4 & 5 \\
\hline \multicolumn{16}{|l|}{ NEFA } \\
\hline EPA iAUC ( $\mu \mathrm{mol} / \mathrm{h}$ per litre $)$ & 31 & 34 & 40 & 33 & 37 & 42 & 33 & 34 & 39 & 30 & 33 & 36 & 32 & 35 & 37 \\
\hline $\mathrm{EPA} C_{\max }(\mu \mathrm{mol} / \mathrm{l})$ & 2 & 3 & 5 & 2 & 4 & 6 & 2 & 3 & 4 & 1 & $2^{*}$ & 3 & 2 & 3 & 4 \\
\hline EPA $T_{\max }(\mathrm{h})$ & 4 & 5 & 6 & 3 & 5 & 6 & 5 & 5 & 6 & 4 & 5 & 6 & 4 & 5 & 6 \\
\hline $\mathrm{DHA}$ iAUC ( $\mu \mathrm{mol} / \mathrm{h}$ per litre) & 45 & 54 & 62 & 53 & 58 & 61 & 52 & 58 & 65 & 55 & 55 & 58 & 51 & 56 & 64 \\
\hline $\mathrm{DHA} C_{\max }(\mu \mathrm{mol} / \mathrm{l})$ & 1 & 4 & 5 & 1 & 4 & 4 & 2 & 4 & 4 & 1 & 2 & 3 & 2 & 3 & 6 \\
\hline $\mathrm{DHA} T_{\max }(\mathrm{h})$ & 4 & 5 & 6 & 2 & 5 & 5 & 4 & 5 & 5 & 4 & 5 & 6 & 4 & 6 & 6 \\
\hline
\end{tabular}

UTAG, unmodified TAG; FFA, free fatty acid; rTAG(EC), enteric-protected re-esterified TAG; EE, ethyl ester; rTAG, re-esterified TAG; iAUC, incremental AUC.

* Median significantly different $(P<0.05)$ from UTAG (Mann-Whitney $U$ test). 
Table 4. Longitudinal changes in EPA and DHA concentrations in plasma TAG, phosphatidylcholine (PC) and NEFA in healthy men (Medians and 25th, 50th and 75th percentiles; $n$ 9/group)

\begin{tabular}{|c|c|c|c|c|c|c|c|c|c|c|c|c|}
\hline \multirow[b]{2}{*}{ Lipid structure of supplements } & \multicolumn{3}{|c|}{ UTAG } & \multicolumn{3}{|c|}{ FFA } & \multicolumn{3}{|c|}{ rTAG(EC) } & \multicolumn{3}{|c|}{ EE } \\
\hline & 25th & 50th & 75th & 25th & 50th & 75th & 25th & 50th & 75th & 25th & 50th & 75th \\
\hline \multicolumn{13}{|l|}{ Plasma lipid class (parameter) } \\
\hline \multicolumn{13}{|l|}{ TAG } \\
\hline EPA iAUC ( $\mu$ mol/week per litre) & 614 & 674 & 762 & 611 & 800 & 877 & 621 & 719 & 798 & 532 & 638 & 748 \\
\hline EPA $C_{\max }(\mu \mathrm{mol} / \mathrm{l})$ & 3 & 10 & 29 & 6 & 15 & 45 & 88 & 15 & 25 & 0 & 4 & 19 \\
\hline EPA $T_{\max }$ (weeks) & 1 & 3 & 8 & 1 & 4 & 6 & 1 & 3 & 8 & 0 & 1 & 12 \\
\hline DHA iAUC ( $\mu \mathrm{mol} /$ week per litre $)$ & 508 & 680 & 706 & 682 & 802 & 888 & 650 & 707 & 797 & 554 & 646 & 709 \\
\hline $\mathrm{DHA} C_{\max }(\mu \mathrm{mol} / \mathrm{l})$ & 3 & 8 & 18 & 8 & 24 & 34 & 8 & 12 & 16 & 0 & 7 & 16 \\
\hline DHA $T_{\max }$ (weeks) & 1 & 3 & 7 & 4 & 8 & 12 & 1 & 5 & 9 & 0 & 1 & 8 \\
\hline \multicolumn{13}{|l|}{ PC } \\
\hline EPA iAUC ( $\mu \mathrm{mol} /$ week per litre) & 808 & 1180 & 1692 & 1470 & 1759 & 2073 & 1231 & 1460 & 1665 & 1225 & 1389 & 1867 \\
\hline EPA $C_{\max }(\mu \mathrm{mol} / \mathrm{l})$ & 73 & 100 & 145 & 120 & 134 & 45 & 99 & 108 & 143 & 99 & 113 & 176 \\
\hline EPA $T_{\max }$ (weeks) & 3 & 4 & 7 & 4 & 8 & 12 & 1 & 2 & 3 & 1 & 2 & 9 \\
\hline DHA iAUC ( $\mu \mathrm{mol} /$ week per litre) & 595 & 1103 & 1458 & 1000 & 1073 & 1280 & 1059 & 1260 & 1395 & 967 & 1284 & 1434 \\
\hline $\mathrm{DHA} C_{\max }(\mu \mathrm{mol} / \mathrm{l})$ & 18 & 44 & 94 & 31 & 39 & 59 & 47 & 60 & 80 & 32 & 50 & 76 \\
\hline DHA $T_{\max }$ (weeks) & 1 & 4 & 7 & 2 & 4 & 10 & 2 & 4 & 8 & 2 & 5 & 9 \\
\hline \multicolumn{13}{|l|}{ NEFA } \\
\hline EPA iAUC ( $\mu \mathrm{mol} /$ week per litre) & 50 & 62 & 77 & 54 & 65 & 84 & 68 & 70 & 77 & 62 & 68 & 84 \\
\hline $\mathrm{EPA} C_{\max }(\mu \mathrm{mol} / \mathrm{l})$ & 1 & 2 & 4 & 2 & 2 & 5 & 3 & 3 & 4 & 2 & 3 & 5 \\
\hline EPA $T_{\max }($ weeks) & 1 & 4 & 11 & 4 & 8 & 12 & 2 & 2 & 9 & 2 & 6 & 12 \\
\hline DHA iAUC ( $\mu \mathrm{mol} /$ week per litre $)$ & 47 & 70 & 82 & 63 & 72 & 100 & 58 & 70 & 79 & 68 & 75 & 90 \\
\hline $\mathrm{DHA} C_{\max }(\mu \mathrm{mol} / \mathrm{l})$ & 1 & 2 & 4 & 1 & 3 & 5 & 2 & 2 & 3 & 2 & 4 & 5 \\
\hline DHA $T_{\max }$ (weeks) & 2 & 6 & 12 & 2 & 4 & 12 & 1 & 2 & 8 & 2 & 4 & 9 \\
\hline
\end{tabular}

UTAG, unmodified TAG; FFA, free fatty acid; $r$ TAG(EC), enteric-protected re-esterified TAG; EE, ethyl ester; iAUC, incremental AUC. 
Table 5. Longitudinal changes in EPA and DHA concentrations in plasma TAG, phosphatidylcholine (PC) and NEFA in healthy women (Medians and 25th, 50th and 75th percentiles; $n$ 9/group)

\begin{tabular}{|c|c|c|c|c|c|c|c|c|c|c|c|c|}
\hline \multirow[b]{2}{*}{ Lipid structure of supplements } & \multicolumn{3}{|c|}{ uTAG } & \multicolumn{3}{|c|}{ FFA } & \multicolumn{3}{|c|}{ rTAG(EC) } & \multicolumn{3}{|c|}{ EE } \\
\hline & 25th & 50th & 75th & 25th & 50th & 75th & 25th & 50th & 75th & 25th & 50th & 75th \\
\hline \multicolumn{13}{|l|}{ Plasma lipid class (parameter) } \\
\hline \multicolumn{13}{|l|}{ TAG } \\
\hline EPA iAUC ( $\mu \mathrm{mol} /$ week per litre) & 522 & 679 & 726 & 626 & 675 & 739 & 300 & 695 & 763 & 412 & 487 & 641 \\
\hline EPA $C_{\max }(\mu \mathrm{mol} / /)$ & 1 & 8 & 21 & 2 & 12 & 27 & 0 & 12 & 32 & 0 & 5 & 8 \\
\hline EPA $T_{\max }$ (weeks) & 1 & 4 & 8 & 1 & 8 & 12 & 0 & 3 & 12 & 0 & 2 & 4 \\
\hline DHA iAUC ( $\mu \mathrm{mol} /$ week per litre) & 543 & 665 & 746 & 577 & 634 & 692 & 426 & 687 & 768 & 383 & 611 & 754 \\
\hline $\mathrm{DHA} C_{\max }(\mu \mathrm{mol} / \mathrm{l})$ & 3 & 11 & 12 & 0 & 0 & 9 & 3 & 12 & 24 & 2 & 4 & 12 \\
\hline DHA $T_{\max }$ (weeks) & 0 & 4 & 13 & 0 & 4 & 12 & 1 & 8 & 12 & 0 & 1 & 3 \\
\hline \multicolumn{13}{|l|}{ PC } \\
\hline EPA iAUC ( $\mu \mathrm{mol} /$ week per litre $)$ & 1650 & 1792 & 1955 & 1125 & 1535 & 2161 & 1220 & 1538 & 2177 & 1403 & 1545 & 2158 \\
\hline EPA $C_{\max }(\mu \mathrm{mol} / /)$ & 140 & 152 & 182 & 76 & 140 & 179 & 93 & 115 & 215 & 114 & 139 & 234 \\
\hline EPA $T_{\max }$ (weeks) & 2 & 4 & 8 & 3 & 4 & 8 & 2 & 8 & 12 & 2 & 4 & 12 \\
\hline DHA iAUC ( $\mu \mathrm{mol} /$ week per litre) & $95 \overline{5}$ & 1185 & 1424 & 911 & 1252 & 1687 & 754 & 1113 & 1425 & 1090 & 1209 & 1415 \\
\hline $\mathrm{DHA} C_{\max }(\mu \mathrm{mol} / \mathrm{l})$ & 39 & 58 & 87 & 27 & 73 & 94 & 14 & 42 & 83 & 32 & 58 & 79 \\
\hline DHA $T_{\max }$ (weeks) & 4 & 4 & 8 & 2 & 4 & 12 & 2 & 8 & 8 & 2 & 4 & 5 \\
\hline \multicolumn{13}{|l|}{ NEFA } \\
\hline EPA iAUC ( $\mu \mathrm{mol} /$ week per litre) & 50 & 62 & 77 & 54 & 65 & 84 & 68 & 70 & 77 & 62 & 68 & 84 \\
\hline $\mathrm{EPA} C_{\max }(\mu \mathrm{mol} / \mathrm{l})$ & 1 & 2 & 4 & 2 & 2 & 5 & 3 & 3 & 4 & 2 & 3 & 5 \\
\hline EPA $T_{\max }$ (weeks) & 1 & 4 & 11 & 4 & 8 & 12 & 2 & 2 & 9 & 2 & 6 & 12 \\
\hline DHA iAUC ( $\mu \mathrm{mol} /$ week per litre $)$ & 47 & 70 & 82 & 63 & 72 & 100 & 58 & 70 & 79 & 68 & 75 & 90 \\
\hline DHA $C_{\max }(\mu \mathrm{mol} / \mathrm{l})$ & 1 & 2 & 4 & 1 & 3 & 5 & 2 & 2 & 3 & 2 & 4 & 5 \\
\hline DHA $T_{\max }$ (weeks) & 2 & 6 & 12 & 2 & 4 & 12 & 1 & 2 & 8 & 2 & 4 & 9 \\
\hline
\end{tabular}

UTAG, unmodified TAG; FFA, free fatty acid; $r$ TAG(EC), enteric-protected re-esterified TAG; EE, ethyl ester; iAUC, incremental AUC. 
no significant differences between supplements in iAUC, $C_{\max }$ or $T_{\max }$ for incorporation of EPA into plasma TAG in men or women (Tables 4 and 5). There was a significant difference between molecular structures of the supplements in the incorporation of DHA into plasma TAG in men (iAUC $\chi^{2}$ $\left(3, n\right.$ 40) $\left.=8.8, P=0.03 ; T_{\max } \chi^{2}(3, n 40)=8.3, P=0.04\right)$, but there was no difference in $C_{\max }$ in men (Table 4 ), and no differences in any of these parameters in women (Table 5). Pairwise comparisons showed that the incorporation of DHA into plasma TAG in men was significantly greater $(122 \mu \mathrm{mol} /$ week per litre, $P=0.02)$, and the peak concentration occurred 4 weeks earlier $(P=0.02)$ for the FFA supplement than for the uTAG, whereas the other supplements did not differ significantly from uTAG.

$T_{\max }\left(\chi^{2}(3, n 40)=10 \cdot 2, P=0 \cdot 02\right)$, but not $C_{\max }$ or iAUC, for incorporation of EPA into plasma PC differed significantly between supplements in men (Table 4), but not women (Table 5). However, this was not supported by pairwise testing. There were no significant differences between supplements in iAUC, $C_{\max }$ or $T_{\max }$ for incorporation of DHA into plasma PC in men or women (Tables 4 and 5).

There were no significant differences between supplements in iAUC, $C_{\max }$ or $T_{\max }$ for incorporation of EPA or DHA into plasma NEFA in men or women (Tables 4 and 5).

There were no significant differences between men and women in EPA or DHA iAUC, $C_{\max }$ or $T_{\max }$ within a supplement molecular structure and plasma lipid class (Tables 4 and 5, online Supplementary Tables S5 and S6). Comparisons between lipid classes showed significant differences in iAUC and $C_{\max }$, but not $T_{\max }$, in postprandial and long-term EPA and DHA incorporation such that $\mathrm{PC}>\mathrm{TAG}>\mathrm{NEFA}$ in both men and women who consumed rTAG(EC), FFA and uTAG (Tables 4 and 5; online Supplementary Tables S5 and S6). However, there was no significant difference in EPA or DHA $C_{\max }$ in men who consumed EE (Table 5; online Supplementary Table S5).

There were no significant associations between BMI or age and the incorporation of EPA or DHA into plasma lipids in men (online Supplementary Table S7). There was no significant association between age and EPA or DHA incorporation into plasma lipids in women. However, there was a significant positive association between BMI and incorporation of EPA and DHA into plasma TAG, but not PC or NEFA, irrespective of lipid structure in women (online Supplementary Table S7).

\section{Discussion}

The overall findings of this research are that the lipid structure of ingested EPA and DHA has, at most, a limited effect on the incorporation of these fatty acids into blood lipids during the postprandial period and during 12 weeks of dietary supplementation. In addition, the incorporation of EPA and DHA into blood lipids during dietary supplementation did not differ between men and women. However, the findings suggest differential incorporation of EPA + DHA into individual plasma lipid classes.

There were inconsistent associations in terms of lipid structure, plasma lipid class and subject sex between BMI and age in the postprandial and supplementation studies. This suggests that neither of these factors was a major determinant of incorporation of EPA and DHA into plasma lipids.

Previous studies have shown that the appearance of EPA and DHA in blood lipids during the postprandial period and during dietary supplementation can be influenced by the lipid structure through which these fatty acids were ingested ${ }^{(10-12)}$, although not all studies have found this ${ }^{(14)}$. The present findings are consistent with these observations in that the peak postprandial concentration of EPA provided as an EE, although not of DHA $\mathrm{EE}$, was approximately half of that of the TAG reference supplement (UTAG) in the plasma NEFA pool. This suggests differential metabolism of EE with fatty acids of different chain length and level of unsaturation, presumably within the gastrointestinal tract, and may reflect differential activity of esterases. In addition, the meal context in which the supplements were consumed may have modified the postprandial metabolism of the $n-3$ fatty acids. For example, consuming EPA + DHA with a high fat meal increased their bioavailability during the postprandial period irrespective of the lipid structure of these fatty acids $^{(11,15)}$. The meal used in the present study contained more than twice as much fat as used in previous studies ${ }^{(5,14)}$, and thus the overall absorption of EPA + DHA would be expected to be greater. It is possible that such an increase in uptake could mask differences in bioavailability between molecular structures of $\mathrm{EPA}+\mathrm{DHA}$ reported in previous studies.

Incorporation of EPA + DHA from rTAG encapsulated in an enteric-resistant coating did not differ significantly from that of gelatin-coated capsules. This suggests that the losses of EPA+ DHA in the stomach are relatively small and that entericprotective coating does not either increase or decrease the bioavailability of EPA + DHA supplements.

There were relatively small, but statistically significant, differences between molecular structures of EPA and DHA regarding their incorporation into blood lipids during the 12 -week supplementation trial. This suggests that longer-term intake of EPA+DHA may have masked any differences between molecular structures seen for acute fatty acid uptake. Previous studies have reported lower omega- 3 index ${ }^{(6,7)}$ and EPA and DHA concentration ${ }^{(21)}$ following consumption of EPA and DHA EE compared with rTAG in patients with hyperlipidaemia or coronary artery disease, respectively. However, three studies in healthy, nomolipidaemic subjects reported that all lipid structures tested were equally effective in increasing EPA and DHA status ${ }^{(13,14,22)}$, although one study reported a pharmaceutical preparation of FFA to be more effective than $\mathrm{EE}^{(9)}$. These findings, together with those of the present study, suggest that differences in the lipid structure of EPA + DHA may have, at most, modest effects on the increase in these fatty acids in blood lipids in healthy individuals. It is possible that the differences between lipid structures reported in some studies $^{(6,7,21)}$ may reflect impaired lipid metabolism associated with dyslipidaemia. In this context, the lipid structure in which EPA and DHA are consumed may have little effect on the increment in status in the general population, both men and women, although this may be an important consideration in specific groups of patients.

Previous studies measured the incorporation of EPA and DHA consumed through different lipid structures either into 
total plasma lipids ${ }^{(9,13,14,23-25)}$ or into single plasma lipid classes $^{(21,26-28)}$. One previous study compared EPA and DHA incorporation into plasma PC and cholesteryl esters during dietary supplementation, but did not report direct comparison between lipid classes ${ }^{(22)}$. Thus, the present findings report for the first time differential incorporation of EPA and DHA into individual plasma lipid classes during both the postprandial period and long-term supplementation. Incorporation of EPA and DHA into plasma PC was greater in terms of iAUC than into plasma TAG and, in turn, into NEFA. The greatest concentration of EPA after a meal was in plasma TAG, compared with plasma $\mathrm{PC}$, and in turn to plasma NEFA. However, this incorporation difference between lipid classes was not found for DHA. Supplementation for 12 weeks resulted in greater concentrations of EPA and DHA in plasma PC when compared with plasma TAG and in turn NEFA. However, the differential plasma lipid pool enrichment may reflect differences in the lipoprotein composition of fasting compared with postprandial blood. Although there was no difference between lipid classes in the time to reach maximum EPA concentration, the appearance of DHA in plasma NEFA was slightly slower than in plasma TAG and PC for some lipid structures. Together, these findings suggest that, as may be expected, EPA and DHA are initially incorporated into plasma TAG and PC, probably in the form of chylomicrons. Appearance in plasma NEFA may reflect incomplete entrapment of fatty acids released by lipoprotein lipase catalysed hydrolysis of chylomicrons ${ }^{(29)}$. However, because the amount of EPA and DHA in each supplement was not equal, it was not possible to assess whether there was differential incorporation of these fatty acids between lipid classes that has been reported previously $^{(30,31)}$

After an initial early postprandial increase in plasma TAG EPA concentration, EPA and DHA were preferentially incorporated into plasma PC compared with TAG or NEFA in both the postprandial period and when consumed as dietary supplements. One possible explanation is that during the postprandial period EPA and DHA from the diet are primarily incorporated into the chylomicron TAG, then released by lipoprotein lipase activity to form NEFA, and subsequently are rapidly incorporated into PC, probably in the liver ${ }^{(31)}$. This is supported by the similar time course of incorporation of EPA and DHA into NEFA and incorporation into TAG and PC, which is consistent with incomplete entrapment of these fatty acids following release by lipoprotein lipase activity ${ }^{(29)}$. These findings are consistent with plasma PC marking long-term EPA and DHA status ${ }^{(32)}$.

The main strengths of this study are that several commercially available EPA and DHA lipid structures were compared directly. In addition, three blood lipid classes were analysed. In the postprandial study, all individuals consumed each of the different types of supplements. One limitation of the postprandial study was that it did not include women. The strength of the dietary supplementation study was that it included both men and women, and thus increased the generalisability of the findings. However, this study had a parallel rather than a crossover design. Other limitations include the exclusion of pregnant women and children who may have different nutritional requirements for EPA and DHA. Pregnant women may also metabolise EPA + DHA differently compared with non-pregnant women because of well-known adaptations to metabolism ${ }^{(33)}$. Furthermore, we did not study the effect of EPA and DHA provided in the phospholipid form. There are claims of enhanced bioavailability of $n-3$ fatty acids provided as phospholipids ${ }^{(26,27)}$. It will be important to explore this using the study designs used in the current research.

Together, these findings show that in healthy individuals neither the lipid structure nor the overall fatty acid composition of supplements that contained EPA and DHA significantly influence their bioavailability during dietary supplementation, despite the apparently lower postprandial bioavailability of $\mathrm{EPA}+\mathrm{DHA}$ EE compared with TAG or FFA. One possible explanation is that any postprandial variation in EPA and DHA bioavailability is lost during longer-term supplementation. Furthermore, there were, at most, small effects of sex on the incorporation of EPA into plasma lipids, which is in agreement with the findings of previous studies ${ }^{(19)}$. One implication of these findings is that choice of the lipid structure of EPA and DHA is not a primary consideration in the design of interventions to improve EPA and DHA status of healthy adults.

\section{Acknowledgements}

This work was supported by a grant from Vifor Pharma, Switzerland. Supplements were donated by Vifor Pharma.

G. C. B. and P. C. C. designed the studies; A. L. W. recruited subjects and conducted both studies including all laboratory work under the supervision of G. C. B. and P. C. C.; A. L. W. and G. C. B. analysed the data; G. C. B. drafted the manuscript; all authors provided intellectual input into the manuscript and agree its content.

P. C. C. serves as an advisor to Pronova BioPharma, Dutch State Mines, Cargill, Smartfish and Danone and has previously served as an advisor to Vifor Pharma and Aker BioMarine. G. C. B. serves as an advisor to BASF, and currently or has previously received research funding from Abbott Nutrition and Nestle. A. L. W. has no conflicts of interest to declare. The funders of the studies (Vifor Pharma) agreed to the design, but had no input into the conduct of the studies, the analysis or interpretation of the data or writing of the manuscript.

\section{Supplementary material}

For supplementary material/s referred to in this article, please visit http://dx.doi.org/doi:10.1017/S0007114516002713

\section{References}

1. Calder PC (2015) Functional roles of fatty acids and their effects on human health. JPEN J Parenter Enteral Nutr 39, 18S-32S.

2. Kris-Etherton PM, Harris WS, Appel LJ, et al. (2002) Fish consumption, fish oil, omega-3 fatty acids, and cardiovascular disease. Circulation 106, 2747-2757.

3. Givens DI \& Gibbs RA (2008) Current intakes of EPA and DHA in European populations and the potential of animal-derived foods to increase them. Proc Nutr Soc 67, 273-280.

4. Dyerberg J, Madsen P, Moller JM, et al. (2010) Bioavailability of marine $n-3$ fatty acid formulations. Prostaglandins Leukot Essent Fatty Acids 83, 137-141. 
5. Harris WS \& Von Schacky C (2004) The Omega-3 Index: a new risk factor for death from coronary heart disease? Prev Med 39, 212-220.

6. Schuchardt JP, Neubronner J, Kressel G, et al. (2011) Moderate doses of EPA and DHA from re-esterified triacylglycerols but not from ethyl-esters lower fasting serum triacylglycerols in statin-treated dyslipidemic subjects: results from a six month randomized controlled trial. Prostaglandins Leukot Essent Fatty Acids 85, 381-386.

7. Neubronner J, Schuchardt JP, Kressel G, et al. (2011) Enhanced increase of omega-3 index in response to long-term $n-3$ fatty acid supplementation from triacylglycerides versus ethyl esters. Eur J Clin Nutr 65, 247-254.

8. el Boustani S, Colette C, Monnier L, et al. (1987) Enteral absorption in man of eicosapentaenoic acid in different chemical forms. Lipids 22, 711-714.

9. Offman E, Marenco T, Ferber S, et al. (2013) Steady-state bioavailability of prescription omega-3 on a low-fat diet is significantly improved with a free fatty acid formulation compared with an ethyl ester formulation: the ECLIPSE II study. Vasc Health Risk Manag 9, 563-573.

10. Lawson LD \& Hughes BG (1988) Human absorption of fish oil fatty acids as triacylglycerols, free acids, or ethyl esters. Biochem Biophys Res Commun 152, 328-335.

11. Davidson MH, Johnson J, Rooney MW, et al. (2012) A novel omega-3 free fatty acid formulation has dramatically improved bioavailability during a low-fat diet compared with omega-3acid ethyl esters: the ECLIPSE (Epanova((R)) compared to Lovaza((R)) in a pharmacokinetic single-dose evaluation) study. J Clin Lipidol 6, 573-584.

12. Wakil A, Mir M, Mellor DD, et al. (2010) The bioavailability of eicosapentaenoic acid from reconstituted triglyceride fish oil is higher than that obtained from the triglyceride and monoglyceride forms. Asia Pac J Clin Nutr 19, 499-505.

13. Laidlaw M, Cockerline CA \& Rowe WJ (2014) A randomized clinical trial to determine the efficacy of manufacturers' recommended doses of omega-3 fatty acids from different sources in facilitating cardiovascular disease risk reduction. Lipids Health Dis $\mathbf{1 3}, 99$.

14. Krokan HE, Bjerve KS \& Mork E (1993) The enteral bioavailability of eicosapentaenoic acid and docosahexaenoic acid is as good from ethyl esters as from glyceryl esters in spite of lower hydrolytic rates by pancreatic lipase in vitro. Biochim Biophys Acta 1168, 59-67.

15. Lawson LD \& Hughes BG (1988) Absorption of eicosapentaenoic acid and docosahexaenoic acid from fish oil triacylglycerols or fish oil ethyl esters co-ingested with a high-fat meal. Biochem Biophys Res Commun 156, 960-963.

16. Burdge GC, Powell J \& Calder PC (2006) Lack of effect of meal fatty acid composition on postprandial lipid, glucose and insulin responses in men and women aged 50-65 years consuming their habitual diets. Br J Nutr 96, 489-500.

17. Folch J, Lees M \& Sloane-Stanley GH (1957) A simple method for the isolation and purification of total lipides from animal tissues. J Biol Chem 226, 497-509.

18. Burdge GC, Wright P, Jones AE, et al. (2000) A method for separation of phosphatidylcholine, triacylglycerol, nonesterified fatty acids and cholesterol esters from plasma by solid-phase extraction. Br J Nutr 84, 781-787.
19. Walker CG, Browning LM, Mander AP, et al. (2014) Age and sex differences in the incorporation of EPA and DHA into plasma fractions, cells and adipose tissue in humans. Br J Nutr 111, 679-689.

20. Caslake MJ, Miles EA, Kofler BM, et al. (2008) Effect of sex and genotype on cardiovascular biomarker response to fish oils: the FINGEN Study. Am J Clin Nutr 88, 618-629.

21. Reis GJ, Silverman DI, Boucher TM, et al. (1990) Effects of two types of fish oil supplements on serum lipids and plasma phospholipid fatty acids in coronary artery disease. Am J Cardiol 66, 1171-1175.

22. Hansen JB, Olsen JO, Wilsgard L, et al. (1993) Comparative effects of prolonged intake of highly purified fish oils as ethyl ester or triglyceride on lipids, haemostasis and platelet function in normolipaemic men. Eur J Clin Nutr 47, 497-507.

23. Ramprasath VR, Eyal I, Zchut S, et al. (2013) Enhanced increase of omega-3 index in healthy individuals with response to 4-week $n$-3 fatty acid supplementation from krill oil versus fish oil. Lipids Health Dis 12, 178.

24. Yurko-Mauro K, Kralovec J, Bailey-Hall E, et al. (2015) Similar eicosapentaenoic acid and docosahexaenoic acid plasma levels achieved with fish oil or krill oil in a randomized double-blind four-week bioavailability study. Lipids Health Dis 14, 99 .

25. Ulven SM, Kirkhus B, Lamglait A, et al. (2011) Metabolic effects of krill oil are essentially similar to those of fish oil but at lower dose of EPA and DHA, in healthy volunteers. Lipids 46, 37-46.

26. Schuchardt JP, Schneider I, Meyer H, et al. (2011) Incorporation of EPA and DHA into plasma phospholipids in response to different omega-3 fatty acid formulations - a comparative bioavailability study of fish oil vs. krill oil. Lipids Health Dis 10, 145.

27. Kohler A, Sarkkinen E, Tapola N, et al. (2015) Bioavailability of fatty acids from krill oil, krill meal and fish oil in healthy subjects - a randomized, single-dose, cross-over trial. Lipids Health Dis 14, 19.

28. Nordoy A, Barstad L, Connor WE, et al. (1991) Absorption of the $n$-3 eicosapentaenoic and docosahexaenoic acids as ethyl esters and triglycerides by humans. Am J Clin Nutr 53, 1185-1190.

29. Evans K, Burdge GC, Wootton SA, et al. (2002) Regulation of dietary fatty acid entrapment in subcutaneous adipose tissue and skeletal muscle. Diabetes 51, 2684-2690.

30. Burdge GC, Sala-Vila A, West AL, et al. (2007) The effect of altering the $20: 5 n-3$ and $22: 6 n-3$ content of a meal on the postprandial incorporation of $n-3$ polyunsaturated fatty acids into plasma triacylglycerol and non-esterified fatty acids in humans. Prostaglandins Leukot Essent Fatty Acids 77, 59-65.

31. Heath RB, Karpe F, Milne RW, et al. (2003) Selective partitioning of dietary fatty acids into the VLDL TG pool in the early postprandial period. J Lipid Res 44, 2065-2072.

32. Browning LM, Walker CG, Mander AP, et al. (2012) Incorporation of eicosapentaenoic and docosahexaenoic acids into lipid pools when given as supplements providing doses equivalent to typical intakes of oily fish. Am J Clin Nutr 96, $748-758$.

33. Herrera E (2000) Metabolic adaptations in pregnancy and their implications for the availability of substrates to the fetus. Eur J Clin Nutr 54, Suppl. 1, S47-S51. 